\title{
The role of gut microbiota in the development of type I diabetes
}

\begin{abstract}
Type 1 diabetes (T1D) is an autoimmune disease characterized by the damage of autoantibody against $\beta$ islet cells. Because of the painful suffering of the patients and the heavy social burdens, T1D has attracted increasing research interests. Several observations made in both rodent models and human patients shed lights on the association between T1D and gut microbiota, yet the underlying mechanism of how microorganisms in the gastrointestinal tract contribute to T1D remain elusive. In the present review we have mainly reviewed recent hypothesis including: (1) interference of gut microbiota with host metabolism and creation of an inflammatory milieu; (2) influence of specific strains or metabolic by-products on the differentiation of host $\mathrm{T}$ cell; and (3) moderation of host gut integrity by certain microbial metabolite. We believe that these knowledge are crucial for the early detection and proper intervention of T1D.

Keywords: type 1 diabetes (T1D), gut microbiota, gut integrity, T cell differentiation
\end{abstract}

Volume 9 Issue 3 - 2018

Dong Yue-xin, Xu Wen-Xie

Basic medical college, Shanghai Jiao Tong University, China

Correspondence: Xu Wen-Xie, Basic medical college, Shanghai Jiao Tong University, Shanghai, 200240, China,

Email wenxiexu@sjtu.edu.cn

Received: March 25, 2018 | Published: May 07, 2018

\section{Introduction}

Gut microbiota is composed of all the microorganisms residing in the gastrointestinal tract. ${ }^{1,2}$ The initial composition of microbiota is affected by how the infant is delivered: during vaginally delivery, newborn infants are exposed to maternal vaginal microbes; while infants delivered by a C-section are exposed to maternal skin microbes. ${ }^{3}$ After that, the microbiota composition is modulated by diet, health status, pharmaceutical agents as well as many other factors., ${ }^{4,5}$ Gut microbes shape the host physiology in various aspects, such as energy homeostasis, immune development and even social behavior.

Several diseases have been linked to gut microbiota, including T1D, an autoimmune disease hallmarked by T-cell recognition of islet antigens and autoimmune-mediated death of insulin-secreting $\beta$ cells. Although incidence of T1D in China is currently low in worldwide comparison ( $\sim 0.1$ cases per 100000 people each year $),{ }^{6}$ the trend of disease incidence is rising rapidly, at least in some area. ${ }^{7}$ Also, considering the large population in China, the absolute quantity of patients suffering T1D is huge. Thus, social and economic burden caused by T1D is large and more attention should be given to this disease.

\section{Gut microbiota and TID}

Various observations made in rodent models indicate that intestinal microbiota might be a contributing factor to T1D. Microbiota composition influences the likelihood of developing T1D. Candon et al. ${ }^{8}$ used antibiotic mixture to completely eliminate gut microbiota and vancomycin to partially eliminate gut microbiota in non-obese diabetic (NOD) mice. In both models, the incidence of T1D rises, with antibiotic mixture showed a stronger effect. Along with these findings, it is reported that mice lacking MyD88, an adapter protein in microbial antigen recognition, did not develop T1D if reared specific pathogen free (SPF), while they developed T1D if kept germ-free (GF). ${ }^{9}$ Moreover, colonization of these GF mice with defined bacteria resembles that in healthy human attenuates T1D. ${ }^{9}$ Microbiota composition of diabetes-prone and diabetes-resistant mice are shown to be different. Using high throughput pyrosequencing and quantitative PCR, Roesch et al. ${ }^{10}$ concluded that Lactobacillus and Bifidobacterium are more abundant in bio-breeding diabetes-resistant (BB-DR) mice, as compared to bio-breeding diabetes-prone (BB-DP) mice.

Intestinal microbiota might also play a role in virus-induced diabetes. Hara and colleagues ${ }^{11}$ reported a transient increase of intestinal Bifidobacterium spp. and Clostridium spp. level after virus injection. This change of microbiota composition can be restored with sulfatrim, which protects the rats from T1D. Other studies focus on the different composition of intestinal microbiota in T1D patients and healthy control. Phylogenetic microarray analysis of intestinal microbiota composition between age-matched children with or without $\mathrm{T} 1 \mathrm{D}^{12}$ concluded that butyrate-producing bacteria predominates the intestinal microbiota in healthy children. In another study, ${ }^{13}$ the same group studied fecal microbial composition in children with or without islet-autoantibody matched for not only age, sex and early feeding history, but also HLA class II genotype, and draw similar conclusions, thus ruling out the possibility that it is the major histocompatability complex genotype which affect the gut microbial composition. Cohort study ${ }^{14}$ also revealed decreased microbiota diversity before the onset of T1D and increase of pro-inflammatory organisms. These experiments collectively indicate the pivotal role of intestinal microbiota in T1D. Next, we will discuss existing mechanisms proposed to elucidate how microorganisms in the gastrointestinal tract contribute to T1D.

\section{Influence production of host metabolites}

Host metabolites can be modified by microbial colonization in mice: ${ }^{15}$ serum phosphatidylcholine (PC) level is dramatically higher in conventionally-raised mice as compared to germ-free mice. Serum PC level has been linked to T1D by a serum metabolome study ${ }^{16}$ showing that children who later developed T1D displayed lower level of phosphatidylcholine at birth. Also, PC is known as an anti-inflammatory molecule. ${ }^{17}$ Therefore, gut microbiota might promote T1D development by eliminating the production of antiinflammatory molecules. Similarly, dysbiosis of gut microbiota 
might contribute to the development of T1D by promoting a proinflammatory intestinal milieu. It has been reported that serum and fecal lysophosphatidylcholines level, a pro-inflammatory molecule, increase prior to the development of disease. ${ }^{14,16}$ These results combined suggest that gut microbiota might contribute to T1D by altering host metabolites.

\section{Gut microbiota interfere with development of host immune system}

\section{Specific bacteria strains modulates $T$ cell differentiation}

Upon different stimulation, the same ancestor, naive $\mathrm{CD} 4^{+}$cells, can differentiate into Th17 cells and T regulatory (Treg) cells. Treg cells suppressed excess immune response, protected against autoimmunity and were marked by the expression of Foxp $3^{18}$ while the role of Th17 cells in T1D was still debatable, as explained in the following paragraphs.

Ivanov et $\mathrm{al}^{19}$ first showed that Th17 cells differentiation in the small intestine required gut microbes and was inhibited by certain antibiotics. Later, Kriegel et al..$^{20}$ studied colonization of segmented filamentous bacterium (SFB) in NOD mice raised in different facilities and concluded a protective effect of SFB in female NOD mice from T1D, but not in their male companions. The underlying mechanism might involve Th17, since SFB have been previously reported to induce naive $\mathrm{CD} 4^{+}$cells differentiation into Th17 cells. ${ }^{21,22}$ Also, it has been reported that the intestinal $\mathrm{T}$ cell antigen receptor is biased toward SBF. ${ }^{23}$ The role of IL-17 producing cells in T1D is controversial, due to the complex nature of various IL-17 producing $\mathrm{T}$ cell subsets. One plausible explanation of its protective effect of Th17 is mediated by suppressed islet-target Th1 effector cells, as in accordance with a previous study. ${ }^{24}$ Though SFB has not been reported as a natural component of human gut microbiota, the possibility that another bacteria fills its gut niche still exist. However, while Kriegel and colleagues as well as other groups $\mathrm{s}^{8,25}$ proposed a protective role of Th17, there are studies that draw opposite conclusion. Hence, the role of SFB in T1D progression needs further inspection. Atarashi et al. ${ }^{26}$ reported that colonization of mice by a certain mix of Clostridium strains resulted in enriched $\mathrm{CD}^{+}$Treg cells, most likely because Clostridium induces the intestinal epithelial cells to release TGF- $\beta$ and other Treg cells-inducing factors.

\section{Metabolic by-products modulate $T$ cell differentiation}

Gut microbes can also affect the development of immune system through their metabolic by-products. They produce short-chain fatty acids (SCFA), including acetate, propionate and butyrate. ${ }^{27}$ Smith et al. ${ }^{18}$ found that germ-free (GF) mice displayed lower level of several important SCFA and they suspected that this might account for the compromised immune system of GF mice, especially reduced size of Treg population. They fed GF mice with SCFA in drinking for three weeks and observed restored Treg size. Further studies agreed with this finding and seek to unveil the underlying mechanisms. Arpaia et al. $^{28}$ reported that butyrate promotes Treg cells differentiation through its histone deacetylase (HDAC) inhibitory activity. Butyrate stabilized acetylation of the Foxp3 locus and increased Foxp3 protein expression, which is crucial for Treg development. They also found that propionate, but not acetate, affect the size and function of Treg cells in the same way. Along with these findings, compositional analysis of the microbiota of T1D children and controls showed that healthy children have more butyrate-producing bacteria in their gut. ${ }^{12,26,29}$
Treg cells play a critical role in immune suppression and their deficiency can leads to T1D, as shown in mice and other animal models. ${ }^{30}$ As for implication in human, Tiittanen et al. ${ }^{31}$ performed quantitative reverse PCR in the small intestinal biopsies from T1D patients and reported comparable density of Foxp3-expression cells with control. A more recent study showed that in T1D patients, while the number of Treg cells was not changed, their function were defective. ${ }^{30}$ Absence of proper autoimmune regulation might contribute to T1D.

\section{Balanced signal hypothesis}

It is proposed that gut microbes can have both pro-inflammatory and anti-inflammatory effect, the host genetics defines the interaction with microbes and the overall outcome should reach a balance. ${ }^{32}$ Burrows et al. ${ }^{33}$ confirmed this theory by showing that Myd88/Trif double knockout NOD mice were not protected from T1D in the presence of conventional microbiota, unlike the NOD single Myd88 knockout. ${ }^{9}$ They proposed that, while Myd88 signaling recognize bacterial antigen and stimulate immune response, TRIF signaling might serve as a bacteria-induced pathway which ameliorate immune response. This hypothesis might explain previous studies that were contradictory about T1D incidence of TLR4 knockout mice, ${ }^{34,35}$ since TLR4 was mediated by both TRIF and Myd88. ${ }^{36}$

\section{Gut microbiota affects intestinal integrity of the host}

Increased gut permeability is linked to T1D in both animal models and human patients. It was reported that gut barrier damage precedes insulitis. ${ }^{37-39}$ Functionally, study of intestinal permeability to sugar in 81 subjects at different stages of T1D and 40 healthy controls concluded that gut integrity is damaged in subjects with islet autoimmunity ${ }^{40}$ In the molecule level, it was reported that genetic susceptible BB mice expressed less tight junction protein claudin and displayed increased intestinal permeability than control. ${ }^{41}$ Morphologically, structure alterations under transmission electron microscopy of intestinal barrier in T1D patients was also observed..$^{39}$

Garcialafuente et $a .^{42}$ excluded a segment of colon from rat, eradicated native microbes with antibiotics and colonized it with specific bacteria strains. They found that certain strains such as Escherichia coli, Klebsiella pneumoniae, and Streptococcus viridans increased the gut permeability to mannitol, whereas other strains such as Lactobacillus brevis decreased gut permeability, providing direct evidence that gut microbes alter intestinal integrity.

Later studies reported similar results, ${ }^{43}$ and the underlying mechanisms have been intensively studied. Using human colonic epithelial cell as experimental subject, Peng et al. ${ }^{44}$ found that butyrate, one of the metabolic by-products of gut microbiota, strength gut barrier by regulating the assembly of tight junction through AMPactivated protein kinase. Brown et al. ${ }^{29}$ also reported butyrate as a key regulator in maintaining gut integrity. Based on metagenomic analysis of stool samples from T1D patients and controls, they proposed a model in which butyrate promotes mucin synthesis, a glycoprotein made by the host to maintain gut barrier, while non-butyrate producing lactate-utilizing bacteria shows opposite effect.

High-fat diet can cause a lipopolysaccharide (LPS)-rich environment in the gut, which has been shown to impair intestinal integrity by interfering the production of tight junction proteins. ${ }^{45}$ This LPS regulating of intestinal barrier was modulated through the 
activation of TLR4 pathway associated with FAK and MyD88. ${ }^{46}$ Prebiotic carbohydrate treatment reduces this high-fat diet-induced dysfunction of gut barrier by a proglucagon-derived peptide (GLP-2)dependent mechanism. ${ }^{47}$

Mucosal-associated invariant T cells (MAIT cells) are a class of innate-like lymphocytes with important roles in microbial defense. Rouxel et al. ${ }^{48}$ suggested in a recent study that MAIT cells can play either a preventive role in T1D by enhancing intestinal barrier function or a pathological role by directly killing $\beta$ islet cells. Switch between preventive and pathological roles could be due to microbiota metabolites presented by $\mathrm{CD} 11 \mathrm{~b}+\mathrm{CD} 11 \mathrm{c}+$ dendritic cells (DCs), sensor of the intestinal environment. ${ }^{49}$

The next question is how this "leaky gut" affect the onset of T1D. It is proposed that the increased gut permeability might allow more exogenous antigen into the mucosal immune system, which further leads to increased production of cytokine that may attack and damage pancreatic beta cells. ${ }^{50}$ Diehl and colleagues ${ }^{51}$ reported that at steady state, intestinal microbes prevents the transportation of both commensal and pathogenic bacteria into mesenteric lymph nodes, and this homeostasis can be affect by antibiotics, which results in $\mathrm{T}$ cell immune response and IgA production. Watts et al. ${ }^{9}$ found that in BB mice, up-regulation of zonulin, which elevates gut permeability by regulating tight junction, precedes autoimmune response against the islet cells. Thus, they concluded that zonulin-related loss of intestinal barrier contributes to T1D development, at least in mice models. Similarly, elevated serum zonulin level correlated with increased gut permeability was observed in T1D patients. ${ }^{52}$

Notably, bacterial metabolites might also attack the islet directly. In mice, streptomyces-derived toxin has been reported to impair glucose tolerance, reduce islet size and decrease relative $\beta$-cell mass at low dose, mainly through its ATPase inhibition. ${ }^{53}$ However, such toxin has yet to be reported in human gut microbiota.

\section{Problems in studies}

These hypothesis are intriguing, yet in need of further proof. While it is widely accepted that intestinal microbes can alter host metabolism, the underlying mechanisms remains unclear. To draw a more definite conclusion about influence of specific bacteria strains on the immune system, longitudinal studies to identify protective or deleterious effect and colonization of defined bacteria species in mice and other animal models might be helpful. To further study the role of Th17 cells in the progression of $\mathrm{T} 1 \mathrm{D}$, method to segregate and recognize $\mathrm{T}$ cell subsets and cytokines should be refined, given the complexity of IL17-expressing cells. While the association between microbiota, "leaky gut" and T1D has attracted much scientific interest, various questions still remain unanswered, such as how a damaged intestinal barrier contributes to autoimmunity.

\section{Summary}

Gut microbiota affects the susceptibility of rodents to T1D. Alteration of the microbial composition by means of antibiotics in turn changes their susceptibility. In human, difference of microbiota composition is observed between T1D patients and healthy control. These findings combined indicate the contributing role of gut microbiota in T1D. Several mechanisms have been proposed to explain the relationship between gut microbiota and T1D: (1) microorganisms residing in the intestine moderate host metabolism, elevating level of either pro- or anti-inflammatory molecules; (2) specific strains or metabolic by-products of the bacteria influence the differentiation of host $\mathrm{T}$ cell; and (3) certain microbial metabolites can either strengthen or reduce host gut integrity, and an impaired intestinal barrier allows more exogenous antigen into the host immune system, leading to increasing cytokines production and $\beta$ cell damage.

The exact mechanisms of how intestinal microbial community affect the onset and progression of T1D are still unclear. We believe that solving these mysteries can not only pave a way for early detection and proper intervention of T1D, but also shed lights on other autoimmune diseases.

\section{Acknowledgements}

None.

\section{Conflict of interest}

The author declares no conflict of interest

\section{References}

1. Qin J, Li R, Raes J, et al. A human gut microbial gene catalogue established by metagenomic sequencing. Nature. 2010;464(7285):5965 .

2. Huttenhower C, Gevers D, Knight R, et al. Structure, function and diversity of the healthy human microbiome. Nature. 2012;486(7402):207-214.

3. Makino H, Kushiro A, Ishikawa E, et al. Mother-to-infant transmission of intestinal bifidobacterial strains has an impact on the early development of vaginally delivered infant's microbiota. PLoS one. 2013;8(11):e78331.

4. Modi SR, Collins JJ, Relman DA, et al. Antibiotics and the gut microbiota. J Clin Invest. 2014;124(10):4212-4218.

5. Jakobsson HE, Jernberg C, Andersson AF, et al. Short-term antibiotic treatment has differing long-term impacts on the human throat and gut microbiome. PLOS ONE. 2010;5(3):e9836.

6. Atkinson MA, Eisenbarth GS, Michels AW. Type 1 diabetes. Lancet. 2014;383(9911):69-82.

7. Zhao Z, Sun C, Wang C, et al. Rapidly rising incidence of childhood type 1 diabetes in Chinese population: epidemiology in Shanghai during 1997-2011. Acta Diabetologica. 2014;51(6):947-953.

8. Candon S, Perezarroyo A, Marquet C, et al. Antibiotics in early life alter the gut microbiome and increase disease incidence in a spontaneous mouse model of autoimmune insulin-dependent diabetes. PLOS ONE. 2015;10(5):e0125448.

9. Wen L, Ley RE, Volchkov P, et al. Innate immunity and intestinal microbiota in the development of Type 1 diabetes. Nature. 2008;455(7216):1109-1113.

10. Roesch LF, Lorca GL, Casella G, et al. Culture-independent identification of gut bacteria correlated with the onset of diabetes in a rat model. ISME J. 2009;3(5):536-548.

11. Hara N, Alkanani AK, Ir D, et al. Prevention of virus-induced type 1 diabetes with antibiotic therapy. Journal of Immunology. 2012;189(8):3805-3814.

12. De Goffau MC, Fuentes S, Den Bogert BV, et al. Aberrant gut microbiota composition at the onset of type 1 diabetes in young children. Diabetologia. 2014;57(8):1569-1577.

13. De Goffau M C, Luopajarvi K, Knip M, et al. Fecal microbiota 
composition differs between children with $\beta$-cell autoimmunity and those without. Diabetes. 2013;62(4):1238-1244.

14. Kostic AD, Gevers D, Siljander H, et al. The dynamics of the human infant gut microbiome in development and in progression toward type 1 diabetes. Cell Host Microbe. 2015;17(2):260-273.

15. Velagapudi V, Hezaveh R, Reigstad C S, et al. The gut microbiota modulates host energy and lipid metabolism in mice. Journal of Lipid Research. 2010;51(5):1101-1112.

16. Oresic M, Simell S, Sysiaho M, et al. Dysregulation of lipid and amino acid metabolism precedes islet autoimmunity in children who later progress to type 1 diabetes. J Exp Med. 2008;205(13):2975-2984

17. Treede I, Braun A, Sparla R, et al. Anti-inflammatory effects of phosphatidylcholine. J Biol Chem. 2007;282(37):27155-27164.

18. Smith PM, Howitt MR, Panikov N, et al. The microbial metabolites, short chainfatty acids, regulate colonic Treg cell homeostasis. Science. 2013;341(6145):569-573.

19. Ivanov II, Frutos Rde L, Manel N, et al. Specific microbiota direct the differentiation of IL-17-producing T-helper cells in the mucosa of the small intestine. Cell Host Microbe. 2008;4(4):337-349.

20. Kriegel MA, Sefik E, Hill JA, et al. Naturally transmitted segmented filamentous bacteria segregate with diabetes protection in nonobese diabetic mice. Natl Acad Sci U S A. 2011;108(28):11548-11553.

21. Ivanov II, Atarashi K, Manel N, et al. Induction of intestinal Th17 cells bysegmented filamentous bacteria. Cell. 2009;139(3):485-498.

22. Gaboriaurouthiau V, Rakotobe S, Lecuyer E, et al. The Key role of segmented filamentous bacteria in the coordinated maturation of gut helper T cell responses. Immunity. 2009;31(4):677-689.

23. Yang Y, Torchinsky MB, Gobert M, et al. Focused specificity of intestinal TH17 cells towards commensal bacterial antigens. Nature. 2014;510(7503):152-156.

24. Oconnor W, Kamanaka M, Booth $\mathrm{C} \mathrm{J}$, et al. A protective function for interleukin 17A in T cell-mediated intestinal inflammation. Nature Immunology. 2009;10(6):603-609.

25. Lau K, Benitez P, Ardissone A N, et al. Inhibition of Type 1 diabetes correlated to a lactobacillus johnsonii N6.2-Mediated Th17 Bias. Journal of Immunology. 2011;186(6):3538-3546.

26. Atarashi K, Tanoue T, Shima T, et al. Induction of colonic regulatory $\mathrm{T}$ cells by indigenous clostridium species. Science. 2011;331(6015):337341

27. Wong JM, De Souza RJ, Kendall CW, et al. Colonic health: fermentation and short chain fatty acids. Journal of Clinical Gastroenterology. 2006;40(3):235-243.

28. Arpaia N, Campbell C, Fan X, et al. Metabolites produced by commensal bacteria promote peripheral regulatory T-cell generation. Nature. 2013;504(7480):451-455.

29. Brown CT, Davis-Richardson AG, Giongo A, et al. Gut microbiomemetagenomics analysis suggests a functional model for the developmentof autoimmunity for type 1 diabetes. PLoS One. 2011;6(10):e25792.

30. Visperas A, Vignali DA. Are regulatory $\mathrm{T}$ cells defective in Type 1 diabetes and can We Fix Them. J Immunol. 2016;197(10):3762-3770.

31. Tiittanen $\mathbf{M}$, Westerholmormio $\mathrm{M}$, Verkasalo $\mathrm{M}$, et al. Infiltration of forkhead box P3-expressing cells in small intestinal mucosa in coeliac disease but not in type 1 diabetes. Clin Exp Immunol. 2008;152(3):498507.
32. Chervonsky A V. Microbiota and autoimmunity. Cold Spring Harbor Perspectives in Biology. 2013;5(3):a007294.

33. Burrows MP, Volchkov $\mathrm{P}$, Kobayashi K S, et al. Microbiota regulates type 1 diabetes through Toll-like receptors. Proc Natl Acad Sci U S A. 2015;112(32):9973-9977.

34. Gulden E, Ihira M, Ohashi A, et al. Toll-like receptor 4 deficiencyaccelerates the development of insulin-deficient diabetes in non-obesediabetic mice. PLoS One. 2013;8(9):e75385.

35. Devaraj S, Tobias P, Jialal I. Knockout of toll-like receptor-4 attenuatesthe pro-inflammatory state of diabetes. Cytokine. 2011;55(3):441-445.

36. Kawai T, Akira S. Toll-like receptors and their crosstalk with other innatereceptors in infection and immunity. Immunity. 2011;34(5):637650 .

37. Graham S, Courtois P, Malaisse W, et al. Enteropathy precedes type 1 diabetes in the BB rat. Gut. 2004;53(10):1437-1444.

38. Meddings J B, Jarand J, Urbanski S J, et al. Increased gastrointestinal permeability is an early lesion in the spontaneously diabetic BB rat. Am J Physiol. 1999;276(4):G951-7.

39. Secondulfo M, Iafusco D, Carratu R, et al. Ultrastructural mucosal alterations and increased intestinal permeability in non-celiac, type I diabetic patients. Digestive and Liver Disease. 2004;36(1):35-45.

40. Bosi E, Molteni L, Radaelli M G, et al. Increased intestinal permeability precedes clinical onset of type 1 diabetes. Diabetologia. 2006;49(12):2824-2827.

41. Neu J, Reverte CM, Mackey A, et al. Changes in intestinal morphology and permeability in the biobreeding rat before the onset of type 1 diabetes. J Pediatr Gastroenterol Nutr. 2005;40(5):589-595.

42. Garcialafuente A, Antolin M, Guarner F, et al. Modulation of colonic barrier function by the composition of the commensal flora in the rat. Gut. 2001;48(4):503-507.

43. Neu J, Lorca G, Kingma SD, et al. The intestinalmicrobiome: relationship to type 1 diabetes. Endocrinol Metab Clin North Am. 2010;39(3):563-571.

44. Peng L, Li Z, Green RS, et al. Butyrate enhances the intestinal barrier by facilitating tight junction assembly via activation of AMP-activated protein kinase in Caco-2 cell monolayers. J Nutr. 2009;139(9):16191625 .

45. Vehik K, Dabelea D. The changing epidemiology of type 1diabetes: why is it going through the roof? Diabetes Metab Res Rev. 2011;27(1):3-13.

46. Cani P, Bibiloni R, Knauf C, et al. Changes in gut microbiota control metabolic endotoxemia-induced inflammation in high-fat diet-induced obesity and diabetes in mice. Diabetes. 2008;57(6):1470-1481.

47. Guo S, Nighot M, Al-Sadi R, et al. Lipopolysaccharide regulation of intestinal tight junction permeability is mediated by TLR4 signal transduction pathway activation of FAK and MyD88. J Immunol. 2015;195(10):4999-5010.

48. Rouxel O, Da JS, Beaudoin L, et al. Cytotoxic and regulatory roles of mucosal-associated invariant $\mathrm{T}$ cells in type 1 diabetes. Nature Immunology. 2017;18(12):1321-1331.

49. Gülden E, Vudattu N K, Deng S, et al. Microbiota control immune regulation in humanized mice. Jci Insight. 2017;2(21)

50. Cani P, Possemiers S, Van de Wiele $\mathrm{T}$, et al. Changes in gut microbiota control inflammation in obese mice through a mechanism 
involving GLP-2-driven improvement of gut permeability. Gut. 2009;58(8):1091-103.

51. Diehl G E, Longman R S, Zhang J, et al. Microbiota restricts trafficking of bacteria to mesenteric lymph nodes by $\mathrm{CX}(3) \mathrm{CR}_{1}$ (hi) cells. Nature. 2013;494(7435):116-120.
52. Sapone A, De Magistris L, Pietzak M, et al. Zonulin upregulation is associated with increased gut permeability in subjects with type 1 diabetes and their relatives. Diabetes. 2006;55(5):1443-1449.

53. Myers M A, Hettiarachchi K D, Ludeman J P, et al. Dietary microbial toxins and Type 1 diabetes. Annals of the New York Academy of Sciences. 2003;1005(1):418-422. 